\title{
Reconstruction of track-type neutrino events with KM3NeT/ORCA
}

\author{
Salvatore Galatà ${ }^{* \dagger}$ \\ APC, Paris \\ E-mail: galata@apc.in2p3.fr
}

KM3NeT is a next-generation research infrastructure being installed in the Mediterranean Sea. Within this infrastructure, KM3NeT/ORCA is a future neutrino detector optimised for the measurement of the neutrino mass hierarchy $(\mathrm{NMH})$ by investigating atmospheric neutrino oscillation in matter in the energy range above $5 \mathrm{GeV}$. Charged-current muon-neutrino events significantly contribute to the NHM sensitivity [1]. In these events, the generated muon induces a track-like topology. The precise reconstruction of such events is an indispensable prerequisite for the NMH measurement. The algorithm described here reconstructs the neutrino direction, the neutrino energy, the position of the interaction vertex and estimates the inelasticity of the neutrino interaction. The performances achieved, in terms of resolutions and efficiency, are presented.

The 34th International Cosmic Ray Conference,

30 July- 6 August, 2015

The Hague, The Netherlands

\footnotetext{
* Speaker.

†n behalf of the KM3NeT collaboration
} 


\section{Introduction}

The topology of a $v_{\mu}$ interaction event in an underwater detector, in the energy range between 5 and $20 \mathrm{GeV}$, strongly depends on the inelasticity (Bjorken-y) of the interaction, which is defined as $\left(1-E_{\mu} / E_{v}\right)$, and indicates how the energy is divided between the hadronic shower at the interaction vertex and the outgoing muon. The two components have very different light patterns that have to be somehow disentangled. The light sources to be accounted for are thus the muon, whose energy loss per unit track length in water is of $0.24 \mathrm{GeV} / \mathrm{m}$, the hadronic shower and the background photons due to the relativistic electrons produced by ${ }^{40} \mathrm{~K}$ decay.

The results presented here are obtained using the official KM3NeT Monte Carlo. Neutrino interactions are simulated using a code based on GENIE. The light production, propagation and detection is simulated using a code based on GEANT that performs the full simulation of the photon propagation. The front-end electronics as well as a realistic and optimised implementation of the trigger have been simulated. A $10 \mathrm{kHz}$ rate of hits due to the decay of ${ }^{40} \mathrm{~K}$ has been chosen, which conservative, considering the value obtained by the latest measurements performed near the foreseen ORCA site [2]. The detector geometry adopted consists of 115 vertical detection units, horizontally separated by $\sim 20 \mathrm{~m}$, with 18 optical modules each, vertically separated by $6 \mathrm{~m}$ along the detection unit, and corresponding to $3.78 \mathrm{Mt}$ of instrumented mass. The footprint of the detector is shown in Figure 1. The optical module simulated is the standard KM3NeT optical module with 31 PMTs of 3" diameter [3]. Since the optimisation of the geometry is still ongoing, this geometry layout is not to be considered as the definitive one. In $\S 6$ the performance of the track reconstruction algorithm is discussed for vertical spacings $\geq 6 \mathrm{~m}$.

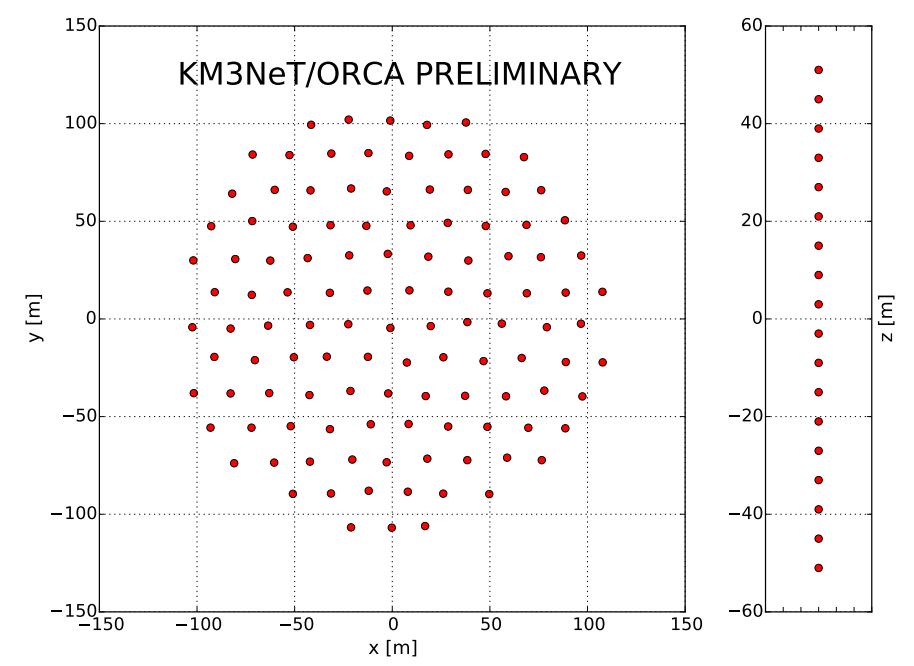

Figure 1: Footprint and vertical displacement of the optical modules on a detection unit of the ORCA detector used in the feasibility study. This detector corresponds to a vertical spacing of $6 \mathrm{~m}$ of the optical modules (right panel), although this quantity is not yet optimised and may change in the future.

The reconstruction algorithm starts by selecting the hits that fulfill space-time coincidence requirements for relativistic particles, as described in $\S 2$, thus reducing the optical background due to the decay of ${ }^{40} \mathrm{~K}$ in the sea water. The muon direction is determined by applying a likelihood 


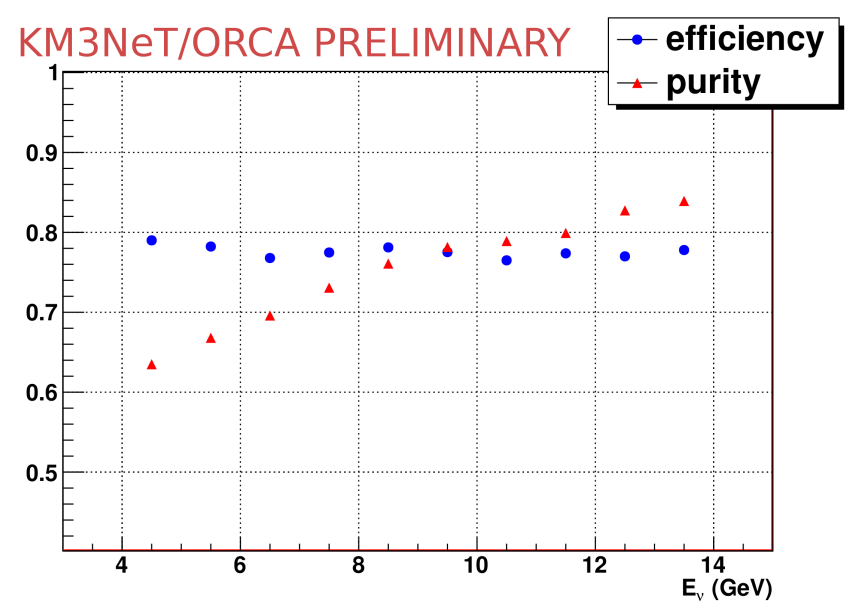

Figure 2: Efficiency and purity of the hit selection adopted by the track reconstruction algorithm as a function of the interacting neutrino energy.

maximisation method based on time residuals with respect to a track hypothesis, which is presented in $\S 3$. The interaction vertex and the muon track length are reconstructed from the time and position of the hits used for the direction reconstruction, as explained in $\S 4$. The Bjorken-y is estimated using the time residuals of the hits with respect to track and cascade hypotheses. The latter is shown in $\S 5$, together with the evaluation of the neutrino energy.

\section{Hit Selection}

Before fitting the track direction, the hits in the event have to be selected in order to reject those which are due to ${ }^{40} \mathrm{~K}$ decay. The selection of the hits is based on the occurrence of local coincidences, i.e. coincidences of hits within the same optical module, as well as on the causality relation between couples of hits. First, all the local coincidences within $10 \mathrm{~ns}$ and for which the PMTs involved do not look more than $90^{\circ}$ apart, are considered. Among them, a cluster is selected such that any hit in the cluster is causally related to all the remaining ones, if the following relation is satisfied:

$$
|\Delta t|<d / c_{\text {water }}+20 \mathrm{~ns}
$$

where $\Delta t$ the time difference between the two hits, $d$ the distance between the two PMTs and $c_{\text {water }}$ is the speed of light in water. The cluster of hits obtained is further extended by including all the other hits which are causally connected and closer than $50 \mathrm{~m}$ to at least $75 \%$ and $40 \%$ of all the hits in the cluster, respectively, and that are all causally connected among them. The latter extension procedure is iterated twice. The resulting performance of the hit selection for $v_{\mu}$-CC events in terms of efficiency and purity is shown in Figure 2, where the efficiency is the fraction of signal hits selected among all the signal hits, whereas the purity is the fraction of signal hits among all the selected ones.

\section{Reconstruction of the neutrino direction}

The reconstruction of the $v_{\mu}\left(\bar{v}_{\mu}\right)$ direction in the case of Charged Current (CC) interactions 


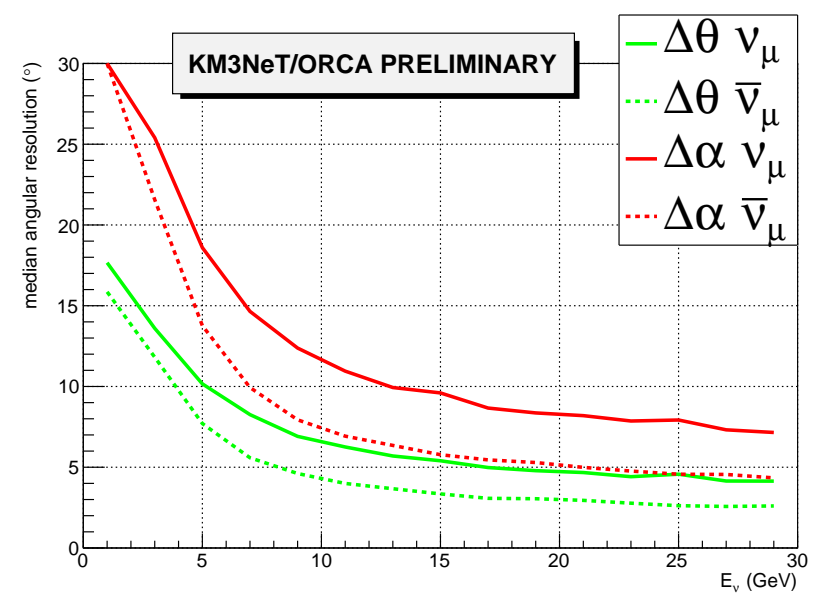

Figure 3: Median resolution of the track reconstruction algorithm on the neutrino angle (red) and zenith (green), for neutrinos (solid) and antineutrinos (dashed).

aims at reconstructing the direction of the outgoing (anti-)muon and is based on a likelihood maximisation algorithm. The likelihood used is based on the distribution of the time residuals, i.e. the difference between the arrival time of the photons at the photomultiplier tubes (PMTs) and the expected arrival time given a certain track hypothesis, which in turn is fully defined by the track direction and its position at a given time. The direction reconstruction is thus fully based on the timing information of the photon hits recorded the PMTs, relying on the favorable properties of the water which, thanks to the relatively long scattering length of optical photons allow for good resolutions to be achieved.

In order to avoid the convergence to local maxima, is it necessary to provide a good starting point to the likelihood maximisation procedure. For this, three preliminary fitting steps are performed in the following order: linear prefit, M-estimator fit, and simplified likelihood fit. By "simplified likelihood" is meant a distribution of the time residuals which does not account for the presence of noise hits due to ${ }^{40} \mathrm{~K}$ decay.

Starting from the track that results from the linear prefit, a scan is performed with a pitch of $3^{\circ}$ in both $\theta$ and $\phi$, for a total of 7200 transformations of the prefit-track, each of which is checked for correlation, in terms of time residuals and distance, with the hit sample obtained in the hit selection procedure described in $\$ 2$. The one hundred most correlated tracks are fitted by both the M-estimator and the simplified likelihood fitter, and the one with the best likelihood is fed to the final likelihood fit. The maximised log-likelihood fitted in the final step is referred to as $\Lambda$ and defines the quality of the reconstruction. Only events with a sufficiently high $\Lambda$ are kept for further analysis and the plots shown in this proceeding imply the application of such a quality cut. The median resolution on the neutrino direction and zenith obtained is shown in Figure 3, separately for neutrinos and anti-neutrinos. The better resolution for antineutrinos with respect to neutrinos is due to the different inelasticity distributions: since for antineutrinos low inelasticity interactions are favoured, this results in higher energy muons which are globally better reconstructed. 


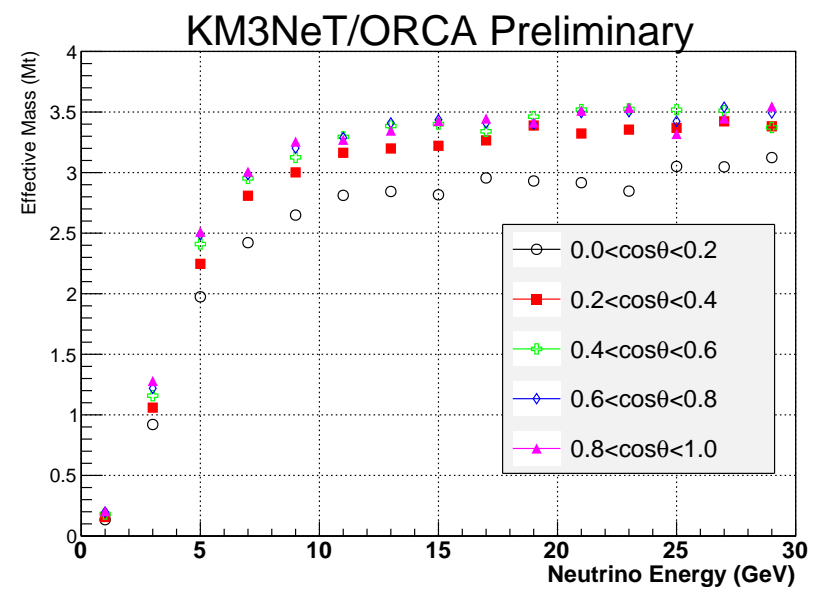

Figure 4: Effective mass for upgoing $v_{\mu}$ 's as a function of the neutrino energy and for steps of $\cos \theta_{v}$ of 0.2 , where $\cos \theta_{v}=0,1$ correspond to horizontal and vertical upgoing events, respectively.

\section{Interaction vertex and muon range estimate}

A dedicated algorithm takes care of estimating the length of the path travelled by the muon within the detector, thus allowing its energy and the interaction vertex to be inferred. First, a subsample of hits (among those obtained using the selection described in $\S 2$ ) related to the muon is selected by requiring the time residuals with respect to the reconstructed track to lie within the interval $-10<t_{\text {res }}(\mathrm{ns})<10$, a maximum orthogonal distance from the reconstructed track of $50 \mathrm{~m}$ and a projected angle of incidence on the PMT $<90^{\circ}$. This strategy selects a very pure set of muon hits. A first estimate of the track length is obtained by back projecting the hits to the direction of the reconstructed track and asking for a minimum local linear density of projected hits of $0.5 \mathrm{~m}^{-1}$. With this procedure, the first back projected hit defines the estimated vertex. This estimate is further refined by performing a dedicated hit selection around the first vertex estimate in the attempt to select the hits associated to the hadronic shower at the interaction vertex, and then using them to perform a 1-dimensional fit along the track direction. The median error on the vertex is $\lesssim 5 \mathrm{~m}$ for $5 \mathrm{GeV}$ neutrinos, and decreases down to $\sim 2 \mathrm{~m}$ at $10 \mathrm{GeV}$.

Only the events reconstructed as upgoing, whose vertex is reconstructed within the instrumented volume and with a sufficienly high reconstruction quality parameter are considered. The corresponding effective mass is show in Figure 4, as a function of the neutrino energy.

\section{Inelasticity and neutrino energy estimation}

The inelasticity of the neutrino interaction is also roughly estimated, since it helps for the further estimation of the total neutrino energy and it may play a role to enhance the overall sensitivity for the measurement of the neutrino mass hierarchy, due to the different inelasticity distribution between neutrino and antineutrino interactions, such that different bins of reconstructed inelasticity would contain different proportions of neutrinos and antineutrinos with increased asymmetry with respect to the asymmetry found in the atmospheric neutrino flux. 


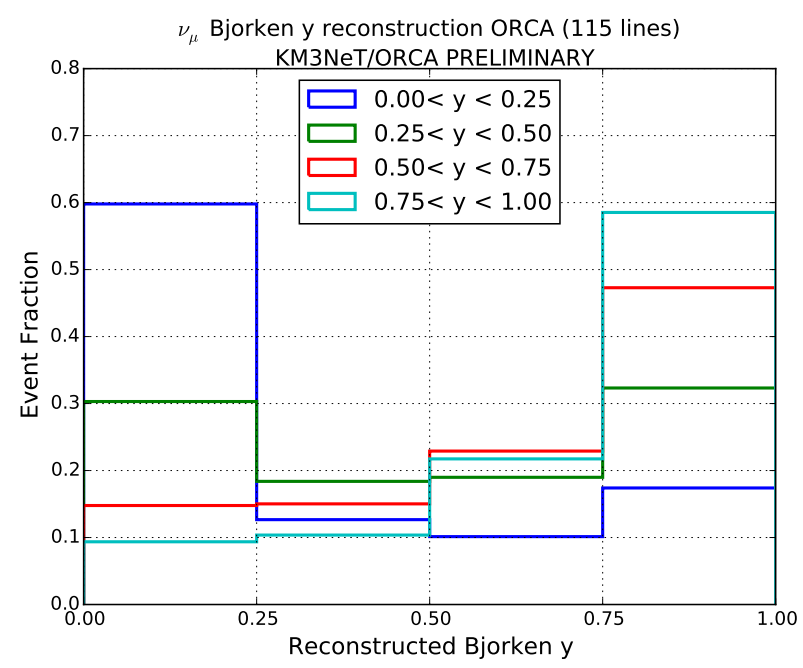

Figure 5: Distribution of the reconstructed Bjorken-y for four bins of true Bjorken-y, indicated by the colour. Each histogram is normalised to 1 .

The Bjorken-y is estimated on the basis of the distribution of the time residuals of the selected hits (cf. § 2) with respect to the reconstructed track and with respect to the reconstructed vertex, according to a track and a shower hypothesis respectively. The rationale for this approach relies on the fact that a different repartition of the total neutrino energy among the hadronic shower and the muon influences the distribution of the time residuals. The estimation is performed among four bins of the Bjorken-y, with a width of 0.25 each. For each bin, the log-likelihood of the time residuals is calculated for a track or shower hypothesis, if the tested Bjorken-y is less or higher than 0.5 , respectively. The Bjorken-y interval corresponding to the highest likelihood is chosen. The performance of the algorithm is shown in Figure 5.

The estimation of the total neutrino energy takes into account the reconstructed muon length, the estimated Bjorken-y and the number of hits used by the reconstruction. Namely, for different values of the reconstructed muon length, the median neutrino energy is fitted as a function of the number of hits used by the reconstruction code. The performance obtained is showed in Figure 6 in terms of the fractional energy resolution, which is defined as $\left|E_{v}-E_{\mathrm{rec}}\right| / E_{v}$.

\section{Performance with an increased vertical spacing}

Although the simulations have been performed with the detector shown in Figure 1, optimisation studies are being carried out to infer the optimal vertical separation of the optical modules within a detection unit. Thus, the performance of the reconstruction algorithm has been studied for vertical spacings $\geq 6 \mathrm{~m}$, in terms of resolutions and efficiency. To perform this study, the detector shown in Figure 1 has been modified by switching off optical modules in order to simulate vertical spacings of $9 \mathrm{~m}$ and $12 \mathrm{~m}$. For the $9 \mathrm{~m}$ configuration, one optical module out of three has been switched off, whereas for the $12 \mathrm{~m}$ configuration one optical module out of two has been switched 


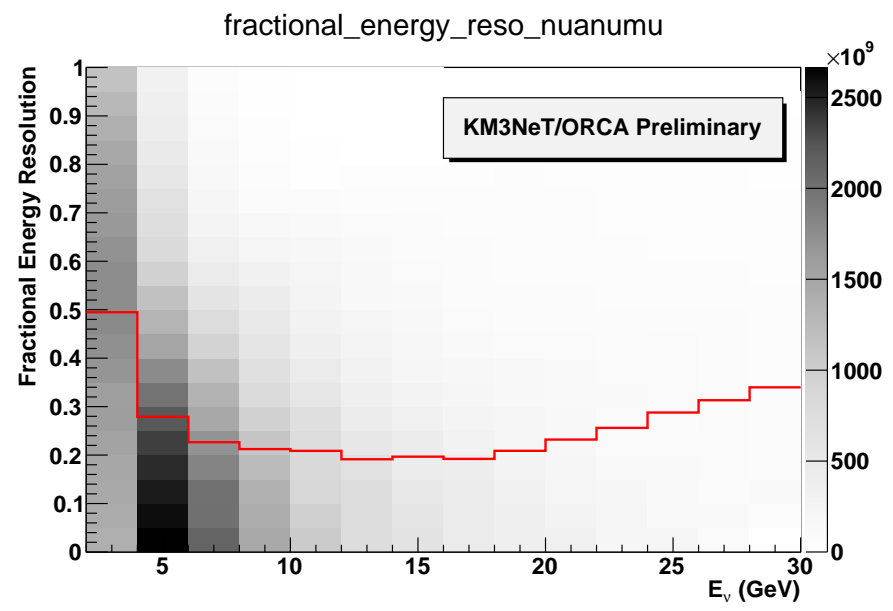

Figure 6: Median fractional energy resolution obtained for $v_{\mu}$ and $\bar{v}_{\mu}$ events.
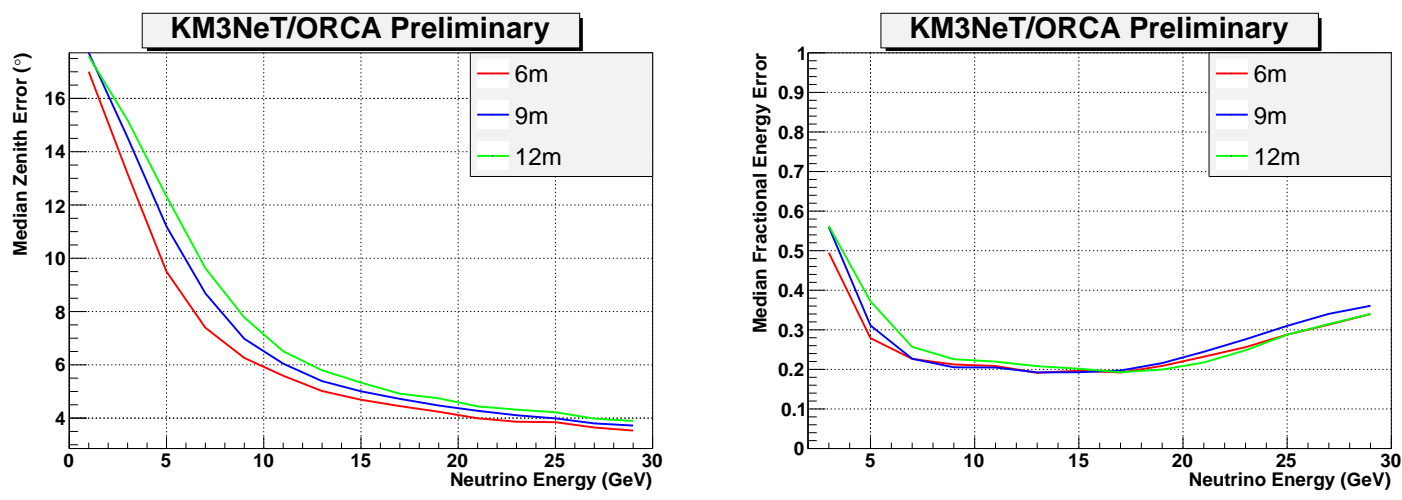

Figure 7: Comparison between the resolutions on the zenith angle (left) and the fractional energy (right) obtained for different vertical spacings of the detector used for the reconstruction. The curves refer to a mix of $v_{\mu}$ and $\bar{v}_{\mu}$ weighted by the atmospheric flux.

off. It is to be noted that the so called $9 \mathrm{~m}$ configuration, indeed alternates vertical spacings of $6 \mathrm{~m}$ and $12 \mathrm{~m}$. With these configurations, the instrumented volume remains unchanged.

The comparison of the zenith resolution and fractional energy resolution obtained for different vertical spacings is shown in Figure 7. The median zenith error degrades by $\sim 1^{\circ}$ at $5 \mathrm{GeV}$ and by $\sim 0.5^{\circ}$ at $10 \mathrm{GeV}$ at every $3 \mathrm{~m}$ step increase of the vertical spacing. The fractional energy resolution slightly deteriorates only below $10 \mathrm{GeV}$.

\section{Conclusions}

The performance of the reconstruction algorithm has been evaluated using a 3.7 Mt detector composed of 115 vertical detection units, horizontally separated by $\sim 20 \mathrm{~m}$, each housing 18 optical modules, vertically separated by $6 \mathrm{~m}$. The neutrino zenith angle is reconstructed with a median error of $\sim 9.5^{\circ}$ at $5 \mathrm{GeV}$ and $\sim 6^{\circ}$ at $10 \mathrm{GeV}$. The median fractional error on the energy is $\sim 0.3$ and $\sim 0.2$ at $5 \mathrm{GeV}$ and $10 \mathrm{GeV}$, respectively. 
The performance has also been evaluated using a geometry that mimics a vertical spacing of $9 \mathrm{~m}$, and one with an exact vertical spacing of $12 \mathrm{~m}$. The median zenith error degrades by $\sim 1^{\circ}$ at $5 \mathrm{GeV}$ and by $\sim 0.5^{\circ}$ at $10 \mathrm{GeV}$ at every $3 \mathrm{~m}$ step increase of the vertical spacing. The fractional energy resolution slightly deteriorates only below $10 \mathrm{GeV}$.

\section{References}

[1] E.Kh. Akhmedov, Soebur Razzaque, and A.Yu. Smirnov. Mass hierarchy, 2-3 mixing and cp-phase with huge atmospheric neutrino detectors. Journal of High Energy Physics, 2013(2), 2013.

[2] Adrián-Martínez et al. Deep sea tests of a prototype of the KM3NeT digital optical module. European Physical Journal C, 74:3056, September 2014.

[3] The KM3NeT Collaboration. Expansion cone for the 3-inch PMTs of the KM3NeT optical modules. Journal of Instrumentation, 8:3006, March 2013. 